\title{
Bone marrow-derived mesenchymal stem cell-conditioned medium attenuates tubulointerstitial fibrosis by inhibiting monocyte mobilization in an irreversible model of unilateral ureteral obstruction
}

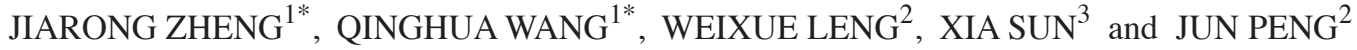 \\ Departments of ${ }^{1}$ Laboratory Medicine and ${ }^{2}$ Nephrology, The People's Hospital of Rizhao; \\ ${ }^{3}$ Department of Pediatrics, Rizhao Central Hospital, Rizhao, Shandong 276826, P.R. China
}

Received October 1, 2017; Accepted February 19, 2018

DOI: $10.3892 / \mathrm{mmr} .2018 .8848$

\begin{abstract}
Mesenchymal stem cell-conditioned medium (MSC-CM) contains various cytokines (osteopontin and macrophage colony stimulating factor 1) secreted by MSCs and may modulate the immune response in tubulointerstitial fibrosis. The aim of the present study was to investigate whether MSC-CM treatment may affect B cell-dependent immune responses, which have previously been reported to facilitate the renal fibrotic processes following unilateral ureteral obstruction (UUO). In the present study, histological analysis, flow cytometry, western blotting and reverse transcription-quantitative polymerase chain reaction were performed. MSC-CM treatment was observed to impede renal infiltration of $\mathrm{B}$ lymphocytes and the expression of $\mathrm{CC}$ chemokine ligand-2. Additionally, UUO suppressed the subsequent recruitment of monocytes/macrophages to the kidney, limited local inflammation and attenuated renal fibrosis. The findings of the present study identified a potential mechanism of MSC-CM in ameliorating the UUO-kidney.
\end{abstract}

\section{Introduction}

As the common end stage of all progressive chronic kidney diseases, renal interstitial fibrosis is defined as an excessive infiltration of leucocytes and deposition of extracellular matrix, leading to the impairment of renal function, destruction of kidney structure and vicious progression to renal failure (1).

Correspondence to: Dr Jun Peng, Department of Nephrology, The People's Hospital of Rizhao, 126 Tai'an Road, Rizhao, Shandong 276826, P.R. China

E-mail:kunkka001@yeah.net

*Contributed equally

Key words: unilateral ureteral obstruction, mesenchymal stem cell-conditioned medium, renal fibrosis, macrophages, B lymphocytes
Research advances regarding this progressive disease have been made; however, patients with end-stage renal disease tend to be dialysis-dependent (2).

Increasing efforts to investigate novel therapeutic strategies to hinder the development of renal interstitial fibrosis have been conducted. In vivo and in vitro studies have suggested that bone marrow-derived mesenchymal stem cell (BMSC)-based therapy produced significant renoprotective effects by reducing renal infiltration of inflammatory cells, glomerulosclerosis and fibrogenesis $(3,4)$. The therapeutic property was mainly attributed to the paracrine effect and immunomodulatory response $(5,6)$; however, allogenic MSC injection may also lead to various problems, including tumorigenesis, maldifferentiation and immune incompatibility (7-10).

Therefore, MSC-conditioned media (MSC-CM), which is rich in cytokines secreted by MSCs, is of primary research interest. Evidence has confirmed the protective effects of MSC-CM in various models $(11,12)$; inhibition of apoptosis, inflammation, cell proliferation and epithelial-mesenchymal transition are all potential mechanisms (9). B cell-dependent immune responses were previously reported to serve a vital role throughout the renal fibrotic process; however, research focusing on the involvement of MSC-CM in this immunoregulatory pattern is limited (13).

In the present study, the model of unilateral ureteral obstruction (UUO) was employed and the therapeutic role of MSC-CM in renal fibrosis was investigated. MSC-CM treatment was demonstrated to attenuate renal fibrosis by hindering B cell-dependent immune responses following UUO in the present study.

\section{Materials and methods}

MSC isolation and preparation of MSC-CM. Primary MSCs were isolated from $\mathrm{C} 57 \mathrm{BL} / 6 \mathrm{~J}$ mice $(\mathrm{n}=60$, male; 8 weeks of age, 20-25 g) by flushing the femurs, and were cultured with Dulbecco's modified Eagle's medium (DMEM; Sigma-Aldrich; Merck KGaA, Darmstadt, Germany) supplemented with $10 \%$ fetal bovine serum (Gibco; Thermo Fisher Scientific, Inc., Waltham, MA, USA). Mice were maintained in air-filtered units at $21 \pm 2^{\circ} \mathrm{C}$ and $50 \pm 15 \%$ relative humidity under a $12 \mathrm{~h}$ 
light/dark cycle during the entire experiment. Mice were provided with rodent food and sterile water ad libitum. MSC-CM was prepared as described previously (14). MSC plates ( $80 \%$ confluence) were incubated with serum-free DMEM low-glucose for $24 \mathrm{~h}$ at $37^{\circ} \mathrm{C}$. Supernatants from each plate were then collected and centrifuged at $500 \mathrm{xg}$ at $4^{\circ} \mathrm{C}$ for $5 \mathrm{~min}$. For each animal, $300 \mu \mathrm{l}$ of conditioned medium generated by approximately $5.0 \times 10^{6}$ cells were injected intraperitoneally (i.p.). The present study was approved by the ethics committee of the People's Hospital of Rizhao (Rizhao, China).

UUO and injection of MSC-CM. All C57BL/6J mice (male, 8 weeks of age) were purchased from the Model Animal Research Center of Nanjing University (Nanjing, China). Mice were divided into four experimental groups: Sham-operated and PBS treated (Sham + PBS), sham-operated and MSC-CM treated (Sham + MSC-CM), UUO model and PBS treated (UUO + PBS), and UUO model and MSC-CM treated (UUO + MSC-CM) mice ( $\mathrm{n}=12$ for each group). Surgical UUO or a sham operation was performed as previously described (15) for UUO and sham groups, respectively. Briefly, the mice were carefully anesthetized using sodium pentobarbital. Following satisfactory anesthesia, a $1 \mathrm{~cm}$ ventral incision was made at the abdominal midline, and the left ureter and kidney were dissociated, and the left proximal ureter was exposed and ligated at two separate locations; sham-operated mice underwent identical exposure without ligation. The incision was then closed using polypropylene sutures, and the mice were allowed to recover from anesthesia. Following surgery, animals received MSC-CM $(300 \mu \mathrm{l})$ or PBS $(300 \mu \mathrm{l})$ i.p., which was repeated 3 days later as previously described $(16,17)$. Flow cytometry and histological assessments were performed at days 3 and 14 following surgery, respectively. Prior to collection of tissue, mice were fasted overnight and anesthetized with $1.5 \%$ pentobarbital sodium $(60 \mathrm{mg} / \mathrm{kg}$ i.p.; Shanghai XiTang Biotechnology Co., Ltd., Shanghai, China). All anaesthetized mice underwent thorax opening and heart exposure. Following the conduction of circulatory system perfusion with heparinized PBS, mice were sacrificed and then the left kidneys were removed for further analysis.

Histological analysis. The left kidney of each mouse was collected and fixed in $10 \%$ formaldehyde as previously described (13). Masson's trichrome and Sirius Red staining procedures (at $25-28^{\circ} \mathrm{C}$ for $20 \mathrm{~min}$ ) were performed on $6 \mu \mathrm{m}$ sections of these paraffin-embedded kidneys for the evaluation of the severity of tubulointerstitial fibrosis. To determine the accumulation of monocytes/macrophages and B lymphocytes, sections of the kidney were also used for immunohistochemical analysis with the following antibodies: Anti-F4/80 (1:200; ab6640; Abcam, Cambridge, UK) and anti-B220 (1:100; ab64100; Abcam) antibodies. Sections were blocked with $5 \%$ bovine serum albumin (Sangon Biotech, Shanghai, China) at $25-28^{\circ} \mathrm{C}$ for $20 \mathrm{~min}$ and then incubated with the primary antibodies overnight at $4^{\circ} \mathrm{C}$. Following incubation with horseradish peroxidase (HRP)-conjugated secondary antibody $\left(1: 1,000\right.$; ab6734; Abcam) at $25-28^{\circ} \mathrm{C}$ for $20 \mathrm{~min}$, sections were incubated with 3,3'-diaminobenzidine. The diaminobenzidine reaction was conducted for $\sim 5 \mathrm{~min}$ at $25-28^{\circ} \mathrm{C}$. For each section, 10 non-consecutive visual fields were randomly selected and captured with a light microscope (magnification, x400, Olympus Corporation, Tokyo, Japan). Images were quantitatively analyzed using Image-Pro Plus 6.0 (Media Cybernetics, Inc., Rockville, MD, USA).

Flow cytometry. Kidney samples were prepared as previously described (13). Briefly, all anaesthetized mice underwent thorax opening. The heart was exposed and a circulatory system perfusion with heparinized PBS was conducted. Renal cortex tissues were then immediately collected, minced and placed into RPMI 1640 medium (Thermo Fisher Scientific, Inc.) containing $40 \mathrm{mg} / \mathrm{ml}$ Liberase TM (Roche Diagnostics, Basel, Switzerland) and 8.5 U/ml DNase I (Roche Diagnostics) for $40 \mathrm{~min}$ at $37^{\circ} \mathrm{C}$. Cells were washed with serum-free RPMI 1640 medium and resuspended in FACS buffer (BD Biosciences, Franklin Lakes, USA) following the addition of red blood cell lysis buffer (BD Biosciences) to exclude erythrocytes. To quantitatively analyze the number of leucocytes, the single cell suspensions were labelled with anti-B220-allophycocyanin (1:100; 17-0452-81; Thermo Fisher Scientific, Inc.) and anti-CD19-phycoerythrin (1:100; 557399; BD Pharmingen; BD Biosciences) for $30 \mathrm{~min}$ in the dark at $4{ }^{\circ} \mathrm{C}$ prior to washing with FACS buffer. Multicolour flow cytometry was performed using a flow cytometer (FACSAriaIII; BD Biosciences) and data was analyzed using FlowJo software version 7.6 (FlowJo LLC, Ashland, OR, USA).

Preparation of $c D N A$ and reverse transcription-quantitative polymerase chain reaction ( $R T-q P C R)$. Total RNA from harvested kidneys was extracted using TRIzol ${ }^{\circledR}$ reagent (Invitrogen; Thermo Fisher Scientific, Inc.) according to the manufactures' protocols. RT was performed using a First-strand cDNA Synthesis kit (Invitrogen; Thermo Fisher Scientific, Inc.) according to the manufacturer's protocols. The PCR cycling conditions were as follows: Predenaturation at $95^{\circ} \mathrm{C}$ for $10 \mathrm{~min}, 40$ cycles at $95^{\circ} \mathrm{C}$ for $15 \mathrm{sec}, 60^{\circ} \mathrm{C}$ for $1 \mathrm{~min}$ and $72^{\circ} \mathrm{C}$ for $20 \mathrm{sec}$, and a final extension at $60^{\circ} \mathrm{C}$ for $5 \mathrm{~min}$. qPCR was performed with Power SYBR ${ }^{\circledR}$ Green PCR Master Mix in a StepOne system (Applied BioSystems; Thermo Fisher Scientific, Inc.). The sequences of the primers for CC chemokine ligand-2 (CCL-2) were as follows: 5'-TTAAAAACCTGG ATCGGAACCAA-3' (forward) and 5'-GCATTAGCTTCA GATTTACGGGT-3' (reverse). Gene expression levels were normalized with GAPDH [5'-GGTGAAGGTCGGTGTGAA CG-3' (forward) and 5'-CTCGCTCCTGGAAGATGGTG-3' (reverse)] and data were quantified and analyzed with StepOne software v2.1 (Thermo Fisher Scientific, Inc.).

Western blotting. Renal tissue samples were used for the extraction of protein and homogenized in ProteoJET Mammalian Cell Lysis Reagent (Fermentas; Thermo Fisher Scientific, Inc.). Total proteins were quantified using the bicinchoninic acid method. Western blotting was performed as previously described (18). Proteins (30 $\mu \mathrm{g} /$ lane) were separated by $10 \%$ SDS-PAGE. The protein was transferred to a polyvinylidene floride membrane and blocked with $5 \%$ skimmed milk for $2 \mathrm{~h}$ at $25-28^{\circ} \mathrm{C}$. Tumor necrosis factor (TNF)- $\alpha(1: 1,000$; ab66579, Abcam), interleukin (IL)-1 $\beta$ (1:1,000; sc-7884, Santa Cruz Biotechnology, Inc., Dallas, TX, USA), intercellular 
A

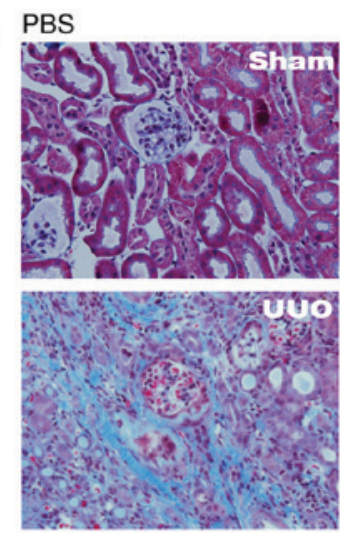

MSC-CM
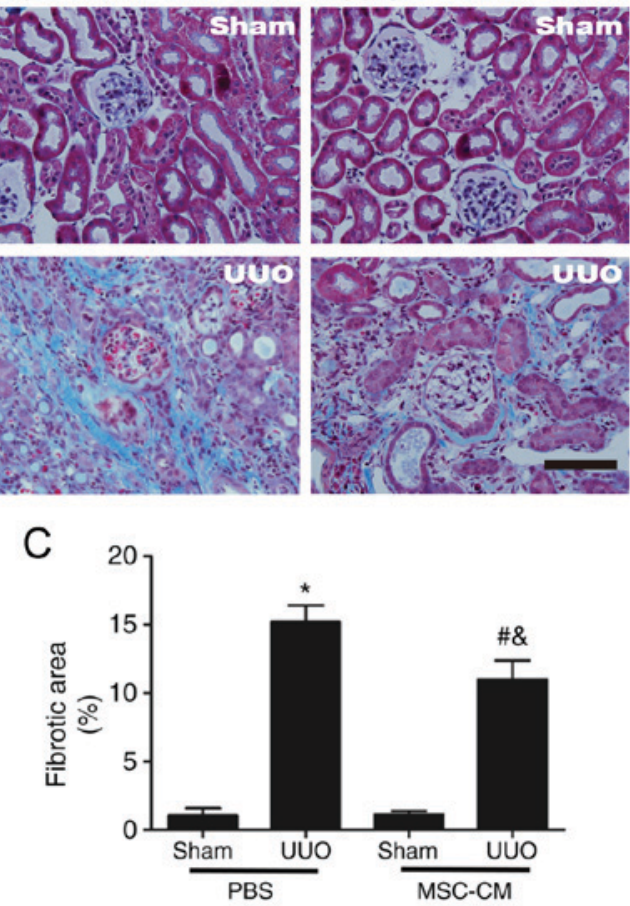

B
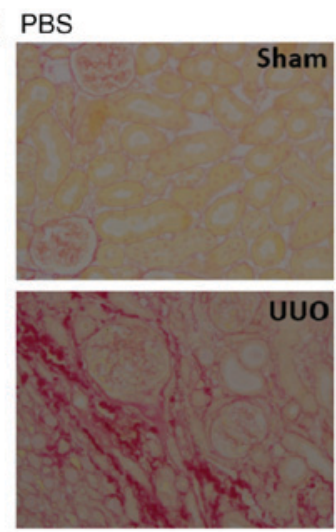

MSC-CM
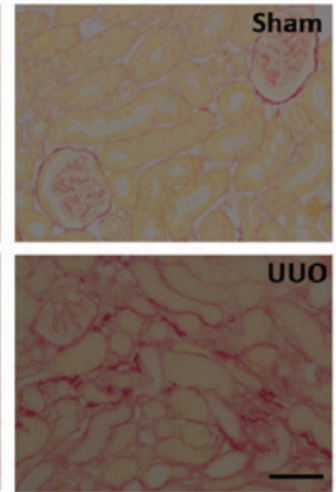

D

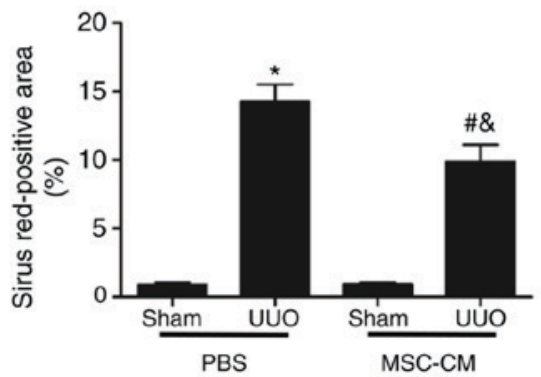

Figure 1. MSC-CM treatment attenuates UUO-induced renal fibrosis. (A) Representative images of renal Masson's trichrome-stained sections in Sham + PBS, Sham + MSC-CM, UUO + PBS, and UUO + MSC-CM mice 2 weeks following surgery. Collagen is visible as the blue color. Scale bar=50 $\mu \mathrm{m}$. (B) Representative images of Sham + PBS, Sham + MSC-CM, UUO + PBS and UUO + MSC-CM mice 2 weeks following surgery. Collagen is visible as the red stain. Scale bar $=50 \mu \mathrm{m}$. (C) Quantitative analysis of renal Masson's trichrome-stained sections. (D) Quantitative analysis of Sirius red-stained renal sections. ${ }^{*} \mathrm{P}<0.05$ vs. Sham + PBS group; ${ }^{\#} \mathrm{P}<0.05$ vs. Sham + MSC-CM group; ${ }^{\&} \mathrm{P}<0.05$ vs. UUO + PBS group. Data are presented as the mean \pm standard deviation ( $\mathrm{n}=6-8)$. MSC-CM, mesenchymal stem cell-conditioned media; Sham + PBS, sham-operated and PBS treated; Sham + MSC-CM, sham-operated and MSC-CM treated; UUO, unilateral ureteral obstruction; UUO + PBS, UUO model and PBS treated; UUO + MSC-CM, UUO model and MSC-CM treated.

adhesion molecule 1 (ICAM-1; 1:1,000; ab179707, Abcam), IL-6 (1:1,000; ab208113, Abcam) were incubated with the membrane overnight at $4^{\circ} \mathrm{C}$. Horseradish peroxidase-labelled goat anti-rabbit secondary antibody $(1: 5,000 ;$ cat. no. 7074 ; Cell Signaling Technology, Inc., Danvers, MA, USA) was added for incubation at room temperature for $1 \mathrm{~h}$, followed by addition of an enhanced chemiluminescence luminous fluid (Beyotime Institute of Biotechnology, Haimen, China) at room temperature for $3 \mathrm{~min}$. The gel was photographed using a gel imaging system. $\beta$-actin (1:2,000; cat. no. 4970; Cell Signaling Technology, Inc.) was used as a house-keeping reference. Densitometric analysis of the western blot results was performed with Image J version 1.48 software (National Institutes of Health, Bethesda, MD, USA).

Statistical analysis. Data were analyzed using SPSS software, version 15.0 for Windows (SPSS Inc., Chicago, IL, USA). Results are presented as the mean \pm standard deviation. The statistical analysis for the determination of differences in the measured properties between groups was accomplished using one-way analysis of variance followed by a Turkey's post hoc test. $\mathrm{P}<0.05$ was considered to indicate a statistically significant difference.

\section{Results}

MSC-CM treatment attenuates UUO-induced fibrosis and renal inflammation. Masson's trichrome and Sirius Red staining was performed to assess fibrotic alterations induced by UUO. The blue-stained (Masson's trichrome-positive) and red-stained (Sirius Red-positive) areas, associated with collagen deposition, were markedly increased in UUO mice compared with sham-operated groups. Notably, treatment with MSC-CM effectively protected the injured kidney from tubulointerstitial fibrosis following UUO, compared with the UUO + PBS group (Fig. 1).

Throughout the procession of UUO-associated fibrogenesis, local inflammatory responses serve a vital role. Therefore, the expression levels of numerous key proinflammatory factors 14 days following the UUO operation were analyzed via western blotting. Statistical analysis revealed that MSC-CM treatment significantly decreased renal expression of TNF- $\alpha$, IL- $1 \beta$, IL- 6 and ICAM- 1 following UUO compared with the UUO + PBS group. In addition, the expression levels of TNF- $\alpha$, IL-1 $\beta$, IL- 6 and ICAM-1 were significantly lower in the sham groups compared with in the UUO groups (Fig. 2).

MSC-CM treatment reduces intrarenal infiltration of monocytes/macrophages following UUO. Monocytes/macrophages are a primary source of proinflammatory factors, and their influx into the renal interstitium is deemed one of the typical features of kidney injury. Analysis of the immunohistochemical results revealed no alterations in the number of infiltrated F4/80-positive cells between PBS and MSC-CM-treated sham mice. Notably, MSC-CM-treated ones revealed a significant 

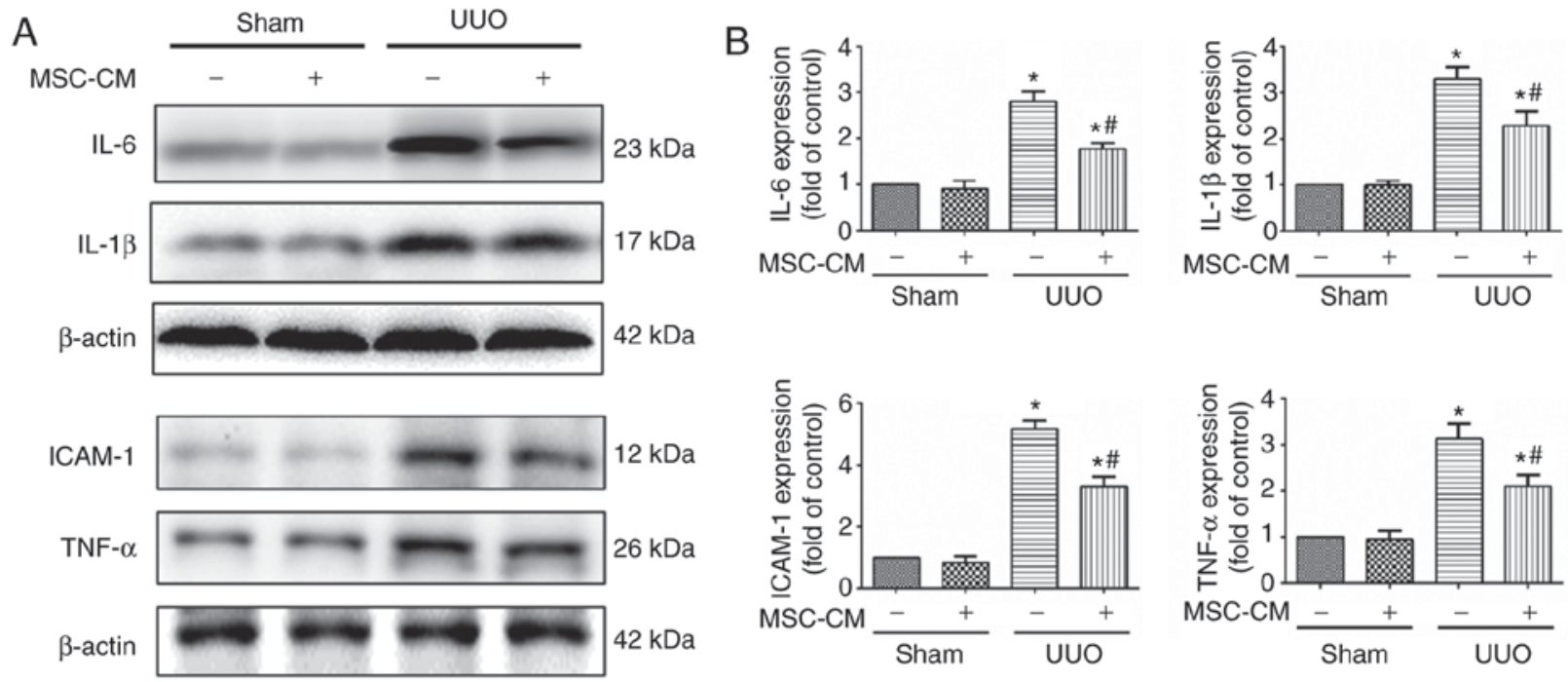

Figure 2. MSC-CM treatment ameliorates UUO-induced renal inflammation. (A) Representative western blotting images and (B) quantitative analysis of the expression levels of key proinflammatory factors in Sham + PBS, Sham + MSC-CM, UUO + PBS, and UUO + MSC-CM mice 3 days following surgery. ${ }^{*} \mathrm{P}<0.05$ vs. Sham + PBS group; ${ }^{\prime} \mathrm{P}<0.05$ vs. UUO + PBS group. ICAM-1, intercellular adhesion molecule 1; IL, interleukin; TNF- $\alpha$, tumor necrosis factor- $\alpha$; MSC-CM, mesenchymal stem cell-conditioned media; Sham + PBS, sham-operated and PBS treated; Sham + MSC-CM, sham-operated and MSC-CM treated; UUO, unilateral ureteral obstruction; UUO + PBS, UUO model and PBS treated; UUO + MSC-CM, UUO model and MSC-CM treated.

A PBS

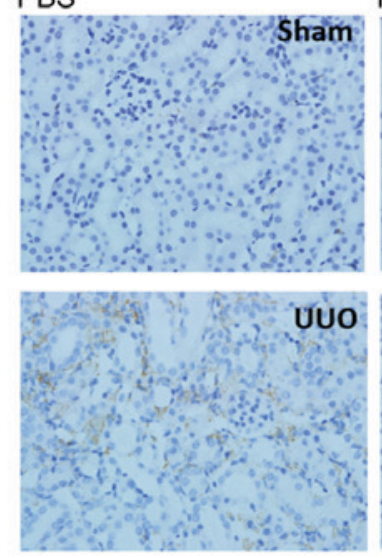

MSC-CM
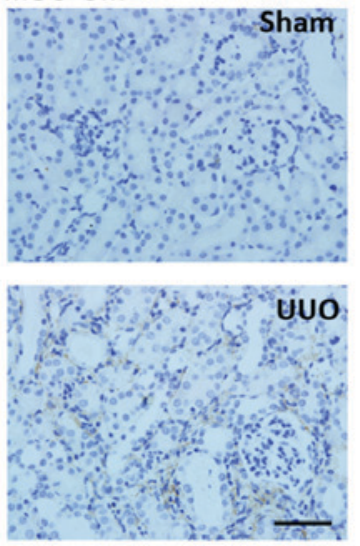

B
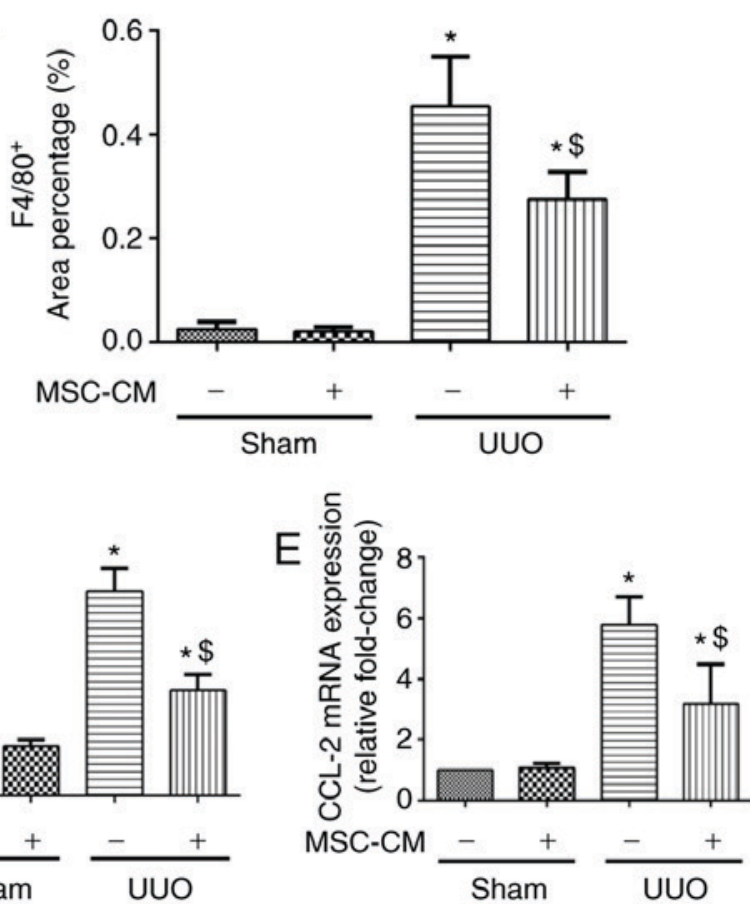

Figure 3. MSC-CM treatment alters chemotactic responses following UUO. (A) Representative images and (B) quantitative analysis of F4/80 staining in Sham + PBS, Sham + MSC-CM, UUO + PBS and UUO + MSC-CM mice 3 days following surgery. Scale bar $=50 \mu \mathrm{m}$. Data are presented as the mean \pm standard deviation (n=7-8). (C) Representative western blotting images and (D) quantitative analysis for CCL-2, 3 days following surgery. Data are presented as the means \pm standard deviation $(n=6)$. (E) Reverse transcription-quantitative polymerase chain reaction analysis of CCL-2 mRNA in kidneys from Sham + PBS, Sham + MSC-CM, UUO + PBS and UUO + MSC-CM mice 3 days following surgery. Data are presented as the means \pm standard deviation $(\mathrm{n}=6) .{ }^{*} \mathrm{P}<0.05$ vs. Sham + PBS group; ${ }^{\$} \mathrm{P}<0.05$ vs. UUO + PBS group. CCL-2, CC chemokine ligand-2; MSC-CM, mesenchymal stem cell-conditioned media; Sham + PBS, sham-operated and PBS treated; Sham + MSC-CM, sham-operated and MSC-CM treated; UUO, unilateral ureteral obstruction; UUO + PBS, UUO model and PBS treated; UUO + MSC-CM, UUO model and MSC-CM treated.

reduction in monocytic infiltration into the kidney 3 days following UUO surgery (Fig. 3A and B).
MSC-CM treatment decreases the expression of local CCL-2 in UUO kidneys. CCL-2, also known as MCP-1, is a member 

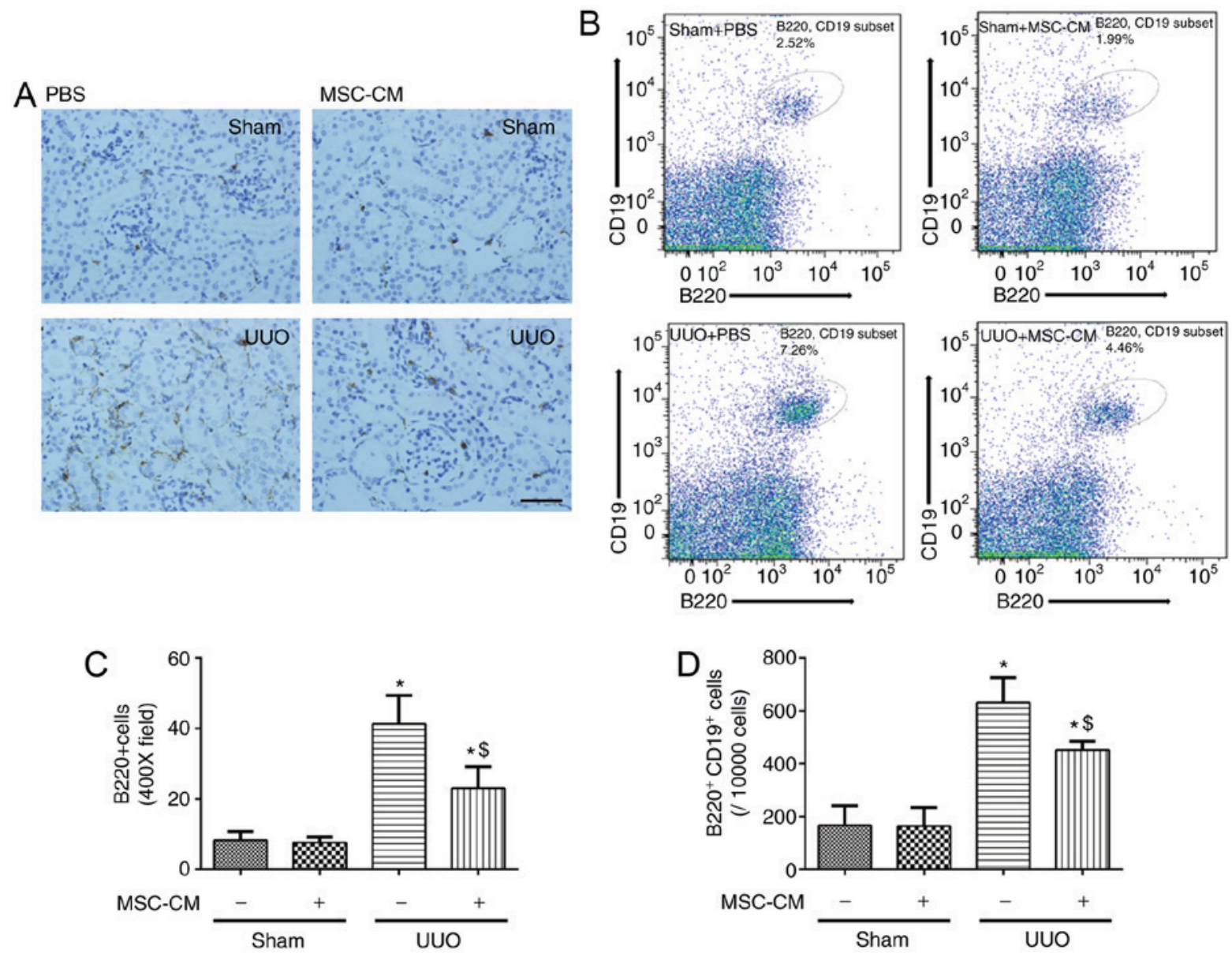

Figure 4. MSC-CM treatment alleviates mature B lymphocyte infiltration into the kidneys following UUO. (A) Representative images of Sham + PBS, Sham + MSC-CM, UUO + PBS and UUO + MSC-CM mice 3 days following surgery. Scale bar=50 $\mu \mathrm{m}$. Data are presented as the mean \pm standard deviation (n=6-7). (B) Representative flow cytometry images of renal B220 ${ }^{+} \mathrm{CD} 19^{+} \mathrm{B}$ lymphocyte cytometry of Sham + PBS, Sham + MSC-CM, UUO + PBS and UUO + MSC-CM mice 3 days following surgery. (C) Quantitative analysis of B220 staining and (D) quantitative analysis of renal B220 $0^{+} \mathrm{CD} 19^{+} \mathrm{B}$ lymphocyte cytometry. Data are presented as the means \pm standard deviation $(n=6) .{ }^{*} \mathrm{P}<0.05$ vs. Sham $+\mathrm{PBS}$ group; ${ }^{\$} \mathrm{P}<0.05$ vs. UUO + PBS group. MSC-CM, mesenchymal stem cell-conditioned media; Sham + PBS, sham-operated and PBS treated; Sham + MSC-CM, sham-operated and MSC-CM treated; UUO, unilateral ureteral obstruction; UUO + PBS, UUO model and PBS treated; UUO + MSC-CM, UUO model and MSC-CM treated.

of CC-family chemokines. Several studies have reported that CCL-2 serves a critical role in the mobilization and relocation of inflammatory cells in response to UUO injury $(13,19,20)$. To investigate the potential mechanisms associated with the effects of MSC-CM, the expression levels of CCL-2 were analyzed via western blotting and RT-qPCR. As revealed by western blotting, UUO resulted in the upregulation of CCL-2, which was attenuated by MSC-CM treatment (Fig. 3C and D). This result was further verified by RT-qPCR (Fig. 3E).

MSC-CM treatment alleviates mature B lymphocyte infiltration into the kidneys following UUO. B lymphocytes $\left(\mathrm{B} 220^{+}\right.$and/or $\mathrm{CD} 19^{+}$cells) are a primary source of renal CCL-2 following UUO, particularly during the early stages. Mice were sacrificed for immunohistochemistry and flow cytometry 3 days following surgery. According to the immunohistochemical analysis, obstructed kidneys suffered greater infiltration of $\mathrm{B} 220^{+} \mathrm{B}$ lymphocytes compared with sham-operated groups. This effect was markedly inhibited by MSC-CM treatment (Fig. 4A and B). Additionally, the results of flow cytometry demonstrated that staining B cells with both anti-B220 and anti-CD19 antibodies, produced a similar effect (Fig. 4C and D).

\section{Discussion}

Previous studies have reported the beneficial effects of MSC-CM administration in modulating renal expression of cytokines associated with inflammation and cell proliferation. Immune cells, including monocytes/macrophages, B and T cells, and mast cells, are also considerable participants in the initiation and progression of renal fibrosis (21-23); however, it is unclear whether MSC-CM exerts an extensive impact on the regulation of intrarenal infiltration of lymphocytes following UUO. The present study revealed a protective effect of MSC-CM in attenuating fibrosis. In this process of treatment, the mobilization of monocytes was efficiently weakened by MSC-CM administration.

Abundant evidence has established that excess inflammation is an essential part of host defense mechanisms following tissue injury (24). By producing various inflammatory cytokines, monocytes/macrophages serve a considerable role in 
the process of post-injury fibrogenesis. Once induced, the progression of the fibrotic process may increase over time, and established fibrosis can seldom be reversed. Therefore, it is a great challenge to intervene from the initial phase and throughout the whole process of the disease. For UUO mice, MSC-CM treatment led to a reduced infiltration of renal monocytes/macrophages, and a decreased expression of pro-inflammatory factors in damaged kidneys, suggesting that MSC-CM may have protected against fibrotic progression by rebalancing the inflammatory response following UUO, particularly at the early stages of pathogenesis.

The reduction of monocytic infiltration and proinflammatory cytokines is most likely a consequence that is associated with the $\mathrm{B}$ cell-mediated immune responses. The present study demonstrated that MSC-CM treatment attenuated the upregulation of CCL-2 induced by UUO. CCL-2, also known as monocyte chemotactic protein 1 , is ubiquitously expressed in a large variety of cell types, including smooth muscle cells, tubular cells, podocytes, mesangial cells and infiltrated leucocytes (25). Via the interaction with CC chemokine receptor-2, CCL-2 mediates the transmigration and influx of inflammatory monocytes into impaired kidneys $(26,27)$. It has previously been demonstrated that early-stage infiltrated B cells are one of the major producers of CCL-2 in the damaged renal tissue (13). The results of the present study revealed that obstructed kidneys exhibited increased incidence of mature B lymphocytic infiltration compared with the sham-operated groups. This effect was significantly alleviated by MSC-CM treatment. These results reinforced the suggestion that MSC-CM may be a potent regulator and option for the treatment of renal immuno-imbalance and interstitial fibrosis following acute injury.

In conclusion, the present study suggested that the administration of MSC-CM ameliorated the intrarenal infiltration of proinflammatory monocytes/macrophages, the level of inflammation associated with renal fibrosis and subsequent fibrotic progression in mice subjected to UUO. The therapeutic effect of MSC-CM may possibly be attributed to the reduced recruitment of B lymphocytes and expression of CCL-2.

\section{Acknowledgements}

We are grateful for the assistance of physicians from the Departments of Laboratory Medicine and Nephrology, the People's Hospital of Rizhao (Rizhao, China).

\section{Funding}

The present study was supported by Discipline Development Fund from the People's Hospital of Rizhao.

\section{Availability of data and materials}

The datasets used and/or analyzed during the current study are available from the corresponding author on reasonable request.

\section{Authors' contributions}

JZ and QW produced the main manuscript text and prepared all the figures and tables. JP participated in the production of the manuscript and contributed to the research design. XS and WL participated in data analysis. All the authors discussed and agreed on the results, and read and approved the final manuscript.

\section{Ethics approval and consent to participate}

The present study was approved by the ethics committee of the People's Hospital of Rizhao (Rizhao, China).

\section{Consent to publication}

Not applicable.

\section{Competing interests}

The authors declare that they have no competing interests.

\section{References}

1. Liu Y: Cellular and molecular mechanisms of renal fibrosis. Nat Rev Nephrol 7: 684-696, 2011.

2. Liu $X$ and Dai C: Advances in understanding and management of residual renal function in patients with chronic kidney disease. Kidney Dis (Basel) 2: 187-196, 2017.

3. Bianco P, Cao X, Frenette PS, Mao JJ, Robey PG, Simmons PJ and Wang CY: The meaning, the sense and the significance: Translating the science of mesenchymal stem cells into medicine. Nat Med 19: 35-42, 2013.

4. Monsel A, Zhu YG, Gennai S, Hao Q, Liu J and Lee JW: Cell-based therapy for acute organ injury: Preclinical evidence and ongoing clinical trials using mesenchymal stem cells. Anesthesiology 121: 1099-1121, 2014.

5. Gnecchi M, Zhang Z, Ni A and Dzau VJ: Paracrine mechanisms in adult stem cell signaling and therapy. Circ Res 103: 1204-1219, 2008.

6. Duffield JS and Bonventre JV: Kidney tubular epithelium is restored without replacement with bone marrow-derived cells during repair after ischemic injury. Kidney Int 68: 1956-1961, 2005.

7. Jeong JO, Han JW, Kim JM, Cho HJ, Park C, Lee N, Kim DW and Yoon YS: Malignant tumor formation after transplantation of short-term cultured bone marrow mesenchymal stem cells in experimental myocardial infarction and diabetic neuropathy. Circ Res 108: 1340-1347, 2011.

8. Kunter U, Rong S, Boor P, Eitner F, Müller-Newen G, Djuric Z, van Roeyen CR, Konieczny A, Ostendorf T, Villa L, et al: Mesenchymal stem cells prevent progressive experimental renal failure but maldifferentiate into glomerular adipocytes. J Am Soc Nephrol 18: 1754-1764, 2007.

9. da Silva AF, Silva K, Reis LA, Teixeira VP and Schor N: Bone Marrow-derived mesenchymal stem cells and their conditioned medium attenuate fibrosis in an irreversible model of unilateral ureteral obstruction. Cell Transplant 24: 2657-2666, 2015.

10. van Koppen A, Joles JA, van Balkom BW, Lim SK, de Kleijn D, Giles RH and Verhaar MC: Human embryonic mesenchymal stem cell-derived conditioned medium rescues kidney function in rats with established chronic kidney disease. PLoS One 7: e38746, 2012.

11. Huang B, Cheng X, Wang H, Huang W, la Ga Hu Z, Wang D, Zhang K, Zhang H, Xue Z, Da Y, et al: Mesenchymal stem cells and their secreted molecules predominantly ameliorate fulminant hepatic failure and chronic liver fibrosis in mice respectively. J Transl Med 14: 45, 2016.

12. Ohnishi S, Sumiyoshi H, Kitamura S and Nagaya N: Mesenchymal stem cells attenuate cardiac fibroblast proliferation and collagen synthesis through paracrine actions. FEBS Lett 581: 3961-3966, 2007.

13. Han H, Zhu J, Wang Y, Zhu Z, Chen Y, Lu L, Jin W, Yan X and Zhang R: Renal recruitment of B lymphocytes exacerbates tubulointerstitial fibrosis by promoting monocyte mobilization and infiltration after unilateral ureteral obstruction. J Pathol 241: 80-90, 2017. 
14. Aslam M, Baveja R, Liang OD, Fernandez-Gonzalez A, Lee C, Mitsialis SA and Kourembanas S: Bone marrow stromal cells attenuate lung injury in a murine model of neonatal chronic lung disease. Am J Respir Crit Care Med 180: 1122-1130, 2009.

15. Nilsson L, Madsen K, Krag S, Frøkiær J, Jensen BL and Nørregaard R: Disruption of cyclooxygenase type 2 exacerbates apoptosis and renal damage during obstructive nephropathy. Am J Physiol Renal Physiol 309: F1035-F1048, 2015.

16. Kay AG, Long G, Tyler G, Stefan A, Broadfoot SJ, Piccinini AM, Middleton J and Kehoe O: Mesenchymal stem cell-conditioned medium reduces disease severity and immune responses in inflammatory arthritis. Sci Rep 7: 18019, 2017.

17. Ooi YY, Dheen ST and Tay SS: Paracrine effects of mesenchymal stem cells-conditioned medium on microglial cytokines expression and nitric oxide production. Neuroimmunomodulation 22 233-242, 2015.

18. Gao F, Wang Y, Li S, Wang Z, Liu C and Sun D: Inhibition of p38 mitogen-activated protein kinases attenuates renal interstitial fibrosis in a murine unilateral ureteral occlusion model. Life Sci 167: 78-84, 2016.

19. Watatani H, Maeshima Y, Hinamoto N, Yamasaki H, Ujike H, Tanabe K, Sugiyama H, Otsuka F, Sato Y and Makino H: Vasohibin-1 deficiency enhances renal fibrosis and inflammation after unilateral ureteral obstruction. Physiol Rep 2: pii: e12054, 2014.

20. Ma W, Tao L, Wang X, Liu Q, Zhang W, Li Q, He C, Xue D, Zhang $\mathrm{J}$ and Liu C: Sorafenib inhibits renal fibrosis induced by unilateral ureteral obstruction via inhibition of macrophage infiltration. Cell Physiol Biochem 39: 1837-1849, 2016.
21. Bohle A, Wehrmann M, Bogenschütz O, Batz C, Vogl W, Schmitt H, Müller CA and Müller GA: The long-term prognosis of the primary glomerulonephritides. A morphological and clinical analysis of 1747 cases. Pathol Res Pract 188: 908-924, 1992.

22. Sean Eardley K and Cockwell P: Macrophages and progressive tubulointerstitial disease. Kidney Int 68: 437-455, 2005.

23. Wynn TA, Chawla A and Pollard JW: Macrophage biology in development, homeostasis and disease. Nature 496: 445-455, 2013.

24. Chevalier RL, Forbes MS and Thornhill BA: Ureteral obstruction as a model of renal interstitial fibrosis and obstructive nephropathy. Kidney Int 75: 1145-1152, 2009.

25. Tesch GH: MCP-1/CCL2: A new diagnostic marker and therapeutic target for progressive renal injury in diabetic nephropathy. Am J Physiol Renal Physiol 294: F697-F701, 2008.

26. Haller H, Bertram A, Nadrowitz F and Menne J: Monocyte chemoattractant protein-1 and the kidney. Curr Opin Nephrol Hypertens 25: 42-49, 2016.

27. O'Connor T, Borsig L and Heikenwalder M: CCL2-CCR2 signaling in disease pathogenesis. Endocr Metab Immune Disord Drug Targets 15: 105-118, 2015.

This work is licensed under a Creative Commons Attribution-NonCommercial-NoDerivatives 4.0 International (CC BY-NC-ND 4.0) License. 